\title{
Curva de aprendizado e lesões iatrogênicas em colecistectomias videolaparoscópicas
}

\section{Learning curve and iatrogenic injuries in laparoscopic cholecystectomies}

Elizabeth Gomes Santos, TCBC-RJ1; Guilherme Pinto Bravo Neto, TCBC-RJ²

R E S U M O

\begin{abstract}
Objetivo: Analisar a proficiência do cirurgião em colecistectomias videolaparoscópicas, de acordo com sua curva de aprendizado, como fator de risco para as lesões iatrogênicas da via biliar principal. Métodos: Estudo retrospectivo de 2285 pacientes submetidos à colecistectomias videolaparoscópicas no Hospital Universitário Clementino Fraga Filho da Universidade Federal do Rio de Janeiro no período de janeiro de 1992 a dezembro de 2007. As características dos cirurgiões foram avaliadas através questionário no qual se procurou identificar idade, experiência profissional em cirurgia convencional e laparoscópica e métodos de aprendizado em videocirurgia. Resultados: O número de lesões da via biliar principal neste estudo foi de seis $(0,26 \%)$, semelhante ao encontrado na literatura mundial. Todas ocorreram na segunda fase da curva de aprendizado, quando os cirurgiões já haviam realizado mais de 50 colecistectomias videolaparoscópicas. Conclusão: Certificado de habilitação, treinamento prévio e idade dos cirurgiões se relacionaram com as lesões da via biliar principal nesta série. Houve significância estatística na diferença entre as médias dos tempos de experiência dos cirurgiões e sua relação com as lesões.
\end{abstract}

Descritores: Doença iatrogênica. Colecistectomia laparoscópica. Cirurgia vídeo-assistida. Aprendizagem. Educação.

\section{INTRODUÇÃO}

$\mathrm{O}$ sucesso de uma operação cirúrgica requer, além de uma indicação precisa, habilidade técnica. Este aprendizado técnico é baseado na repetição de movimentos e passa por uma fase na qual o aprendiz ganha competência rapidamente para, em seguida, atingir um platô, após o qual não se observa nenhuma modificação no ganho de habilidades, embora continue a ganhar velocidade.

Desde sua primeira realização bem sucedida em 1987, a Colecistectomia Videolaparoscópica (CVL) tornouse um dos procedimentos cirúrgicos mais realizados no mund $^{1-3}$. No Brasil foi realizada pela primeira vez em 1990 em São Paulo. No Hospital Universitário Clementino Fraga Filho da Universidade Federal do Rio de Janeiro (HUCFFUFRJ) a primeira CVL foi feita em 1992.

Entretanto, mesmo proporcionando aos pacientes todas as vantagens já conhecidas, muitas publicações mostraram grande aumento nos índices de lesão iatrogênica da VBP, com variações entre $0,16 \%$ a $2,36 \%$ em algumas séries para os primeiros casos contra os $0,07 \%$ a $0,95 \%$ das colecistectomias por laparotomia ${ }^{4-6}$. Os principais motivos apontados para explicar a maior incidência destas lesões foram a presença de reação inflamatória intensa, variações anatômicas do pedículo biliar, sangramento duran- te a operação, uso indiscriminado do termocautério, a qualidade do equipamento e, a mais importante de todas, a inexperiência do cirurgião com o novo método ${ }^{7-9}$.

Observou-se que, com o desenvolvimento da habilidade e ganho de experiência, estes índices tornavamse menores e comparáveis aos dos procedimentos chamados convencionais. Surgiram assim, estudos que objetivaram relacionar a curva de aprendizado do cirurgião com os índices de lesões e o estágio da curva no qual o cirurgião teria experiência suficiente para evitá-las.

\section{MÉTODOS}

Análise retrospectiva dos dados de pacientes submetidos à CVL realizadas no HUCFF-UFRJ no período compreendido entre janeiro de 1992 e dezembro de 2007, assim como dos cirurgiões do Serviço de Cirurgia Geral do HUCFF- UFRJ envolvidos com videocirurgia. Todos os pacientes foram operados eletivamente e a indicação da operação foi a colelitíase sintomática. Projeto submetido ao Comitê de Ética em Pesquisa do HUCFF-UFRJ número 122/08.

Foram analisadas as lesões iatrogênicas do ducto hepático comum e de seus ramos e do colédoco, reconhecidas no intra-operatório ou no pós-operatório até trinta

Trabalho realizado no Hospital Universitário Clementino Fraga Filho - Universidade Federal do Rio de Janeiro - UFRJ- Rio de Janeiro - RJ-BR. 1. Cirurgiã do Serviço de Cirurgia Geral do Hospital Universitário Clementino Fraga Filho - UFRJ - Rio de Janeiro - RJ - BR; 2. Professor Adjunto do Departamento de Cirurgia da Faculdade de Medicina - UFRJ - Rio de Janeiro - RJ - BR. 
dias. Para o estudo destas lesões foi utilizada a classificação de Csendes $^{1}$. (Tabela 1) que trata das lesões agudas da VBP durante CVL.

A experiência do cirurgião como fator de risco para lesões da via biliar principal (VBP) teve maior ênfase neste estudo. Foram também considerados a presença de processo inflamatório crônico intenso, sangramento intraoperatório de difícil controle, variações anatômicas das vias biliares extra-hepáticas, idade avançada, e a doença biliar no sexo masculino.

Os cirurgiões foram distribuidos em dois grupos: grupo A - cirurgiões que tiveram pacientes com lesões da VBP e grupo B - cirurgiões que não tiveram pacientes com lesões da VBP. Dados como idade, tempo global de prática, treinamento prévio em videocirurgia, experiência em videocirurgia e a quantidade de CVL já realizadas, foram coletados e analisados através de questionário enviado a 22 cirurgiões

A análise da experiência dos cirurgiões foi baseada na curva de aprendizado para CVL. Para a primeira fase da curva considerou-se um número mínimo de $50 \mathrm{CVL}$ realizadas como cirurgião principal. A partir daí iniciava-se a segunda fase da curva, em que o cirurgião foi considerado proficiente para realização do procedimento.

Para análise estatística foram utilizados os coeficientes de associação Phi e o P de Person para avaliação da relação entre certificado de habilitação, treinamento em videocirurgia e idade do cirurgião como fator de risco e lesão.

Para avaliar a relação entre a experiência prévia em cirurgia, média de CVL por ano, tempo médio de experiência em videocirurgia e lesão foi utilizado o Teste t de student para a testar a hipótese de igualdade das médias.

\section{RESULTADOS}

No período estudado foram realizadas 2.285 CVL conforme distribuição mostrada na figura 1. A maioria dos pacientes (85\%) era do sexo feminino e $15 \%$ do masculino. A média da idade dos pacientes foi de 48 anos (mínimo de 12 e máximo de 95). A queda do número de colecistectomias deveu-se à problemas estruturais do HUCFF - UFRJ a partir de 2003.

Dos 22 cirurgiões praticantes de videocirurgia para os quais foi enviado o questionário, apenas um não respondeu.

Neste período ocorreram seis lesões da VBP, um índice de 0,26\%. Estas lesões ocorreram com cinco dos 21 cirurgiões e um dos cirurgiões foi responsável por duas lesões. Das lesões encontradas, duas foram do ducto hepático direito, tipo I de Csendes, uma delas por incisão lateral causada pela inserção de cateter para colangiografia em vesícula séssil inserindo-se no ducto hepático direito e outra por provável lesão térmica. A terceira tratou-se de lesão da junção cístico-coledociana em vigência de inflamação na área do pedículo, classificada como tipo II de Csendes. Das outras três lesões, uma foi causada por ligadura e secção completa do ducto hepático comum e as duas outras envolveram ligadura, secção completa e ressecção de segmento da VBP, tipos III e IV de Csendes respectivamente.

Outros fatores de risco também presentes nos pacientes que tiveram lesões foram: processo inflamatório crônico intenso em três casos (50\%), sangramento intraoperatório de difícil controle em um caso $(16,66 \%)$, variações anatômicas em dois (33,33\%), idade avançada em três $(50 \%)$ e sexo masculino em dois $(33,33 \%)$ Quatro pacientes $(66,66 \%)$ apresentavam dois fatores de risco, e em um $(16,66 \%)$ foram identificados três fatores de risco. Em um caso apenas $(16,66 \%)$ não havia nenhum fator de risco identificável. Quatro lesões $(66,66 \%)$ foram diagnosticadas no intra-operatório e tratadas no mesmo ato cirúrgico e pelo mesmo cirurgião. As outras duas $(33,33 \%)$ foram identificadas em pacientes com quadro de abdômen agudo por peritonite no pós-operatório, com 48 e 72 horas, respectivamente.

\section{Colecistectomias}

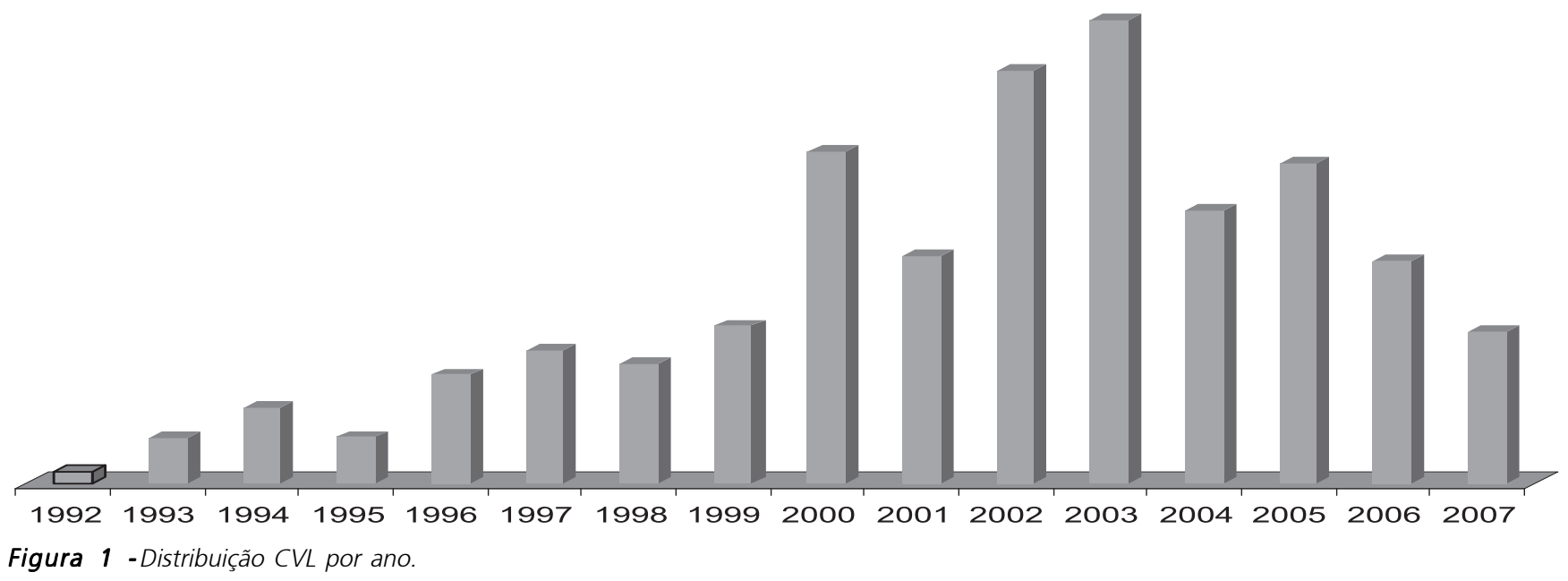


Tabela 1 - Classificação de Csendes.

\begin{tabular}{ll}
\hline Tipo & Descrição \\
\hline I & $\begin{array}{l}\text { Pequena laceração do hepático comum ou do ramo } \\
\text { hepático direito }\end{array}$ \\
II & Lesão da junção cístico-coledociana \\
III & Secção ou ligadura parcial ou completa da VBP \\
IV & Secção com ressecção de mais de $10 \mathrm{~mm}$ da VBP \\
\hline
\end{tabular}

Todas as lesões ocorreram em pacientes operados por cirurgiões que já haviam realizado mais de 50 colecistectomias e estavam, portanto, na segunda fase da curva de aprendizado.

A colangiografia, indicada de modo seletivo, foi realizada nesta série em $6,45 \%$ dos casos. Naqueles em que houve lesão ela foi realizada em $66,66 \%$. Nos pacientes com lesão descoberta no mesmo ato operatório, o tempo médio de internação foi 11 dias, variando entre quatro e 15. O tempo de seguimento após a alta dos pacientes com lesão variou de quatro a 168 meses. Não houve óbitos decorrentes destas lesões.

$\mathrm{Na}$ análise estatística, o coeficiente Phi foi confirmado pelo coeficiente de Pearson e mostrou que houve baixa associação entre a habilitação em videocirurgia, o treinamento, a idade do cirurgião e o índice de lesões para os dois grupos de cirurgiões. Quando foi aplicado o Teste t de student, este mostrou que o grupo $\mathbf{B}$ tinha o tempo médio de experiência significantemente menor do que o do grupo $A$ e que não houve diferença significativa entre os grupos $\mathbf{A}$ e $\mathbf{B}$ em relação à média de videocirurgias cirurgias realizadas por ano e a experiência prévia em videocirurgia e o índice de lesões.

\section{DISCUSSÃO}

A lesão iatrogênica da VBP é a complicação mais grave da CVL e a secção com ressecção de segmento do ducto biliar, acompanhada ou não de lesão vascular, a mais grave de todas $^{10}$. Connor e Garden ${ }^{11}$ afirmam que estas lesões devem ser vistas como complicações evitáveis, embora na opinião de mais de $70 \%$ dos cirurgiões por eles entrevistados, mesmo com a observância de todas as medidas profiláticas elas são inevitáveis. Francoeur et $a^{\beta}$ acreditam que um em cada dois cirurgiões, mesmo os mais experientes, será responsável por uma lesão em algum momento de sua carreira.

O acúmulo de conhecimentos decorrentes da prática, que determinam o ganho de experiência sofreu grandes mudanças com a videocirurgia passando-se a valorizar a experiência do cirurgião com base na curva de aprendizado com o método laparoscópico. A Sociedade Brasileira de Videocirurgia ${ }^{12}$ estabelece em 25 o número mínimo de procedimentos realizados, para que o cirurgião possa requerer o Certificado de Habilitação. Já o Colégio Brasileiro de Cirurgiões ${ }^{13}$ em associação com o Colégio Brasileiro de Cirurgia Digestiva e com a Sociedade Brasileira de Coloproctologia, entre as várias etapas do concurso para obtenção do Certificado de Habilitação, exige participação em cursos teórico-práticos e comprovação de 15 intervenções videolaparoscópicas.

Inicialmente pensou-se que o aumento da incidência das lesões iatrogênicas da VBP estava relacionado ao processo de aprendizado da nova tecnologia. Estudos recentes, no entanto, revelaram que os índices de lesão são ainda grandes mesmo após tanto tempo decorrido da primeira CVL, e maiores em algumas séries quando comparados aos da colecistectomia por laparotomia. Além disto, estas lesões são também mais graves ${ }^{14,15}$ e ocorrem a despeito da experiência, da habilidade e do treinamento do cirurgião ${ }^{4,16,17}$

A curva de aprendizado é definida na prática, como o número de repetições necessário para que o aprendiz desenvolva a habilidade e atinja a proficiência, que é o momento em que o cirurgião pode realizar o procedimento sem supervisão ${ }^{18}$. Segundo Melo ${ }^{19,20}$ ao se realizar treinamento em videocirurgia, é necessária a aprendizagem de habilidades psicomotoras indispensáveis ao método laparoscópico. A coordenação olho-mão é mais rapidamente desenvolvida em indivíduos mais jovens e representa um fator facilitador na aquisição de habilidades motoras necessárias à videocirurgia ${ }^{19-21}$. A habilidade requerida para a cirurgia pode ser adquirida através de um número variável de horas de treinamento, que somado ao conhecimento da anatomia e da técnica operatória, tornam o cirurgião capacitado à realização de operações cada vez mais complexas $^{20}$

A aprendizagem se faz através de três áreas distintas: a cognitiva, relacionada ao conhecimento, a afetiva, associada aos sentimentos e a psicomotora que é relacionada ao fazer propriamente dito e que se traduz pelas ações de natureza física ${ }^{21-23}$. A capacidade de aprender difere entre os indivíduos, mas obedece a um mesmo padrão entre seres humanos que já atingiram a maturidade, e só é influenciada negativamente pela a idade quando as enfermidades deterioraram as áreas da aprendizagem afetando em especial o domínio psicomotor ${ }^{14,19,20}$.

A qualidade do desempenho do cirurgião muda ao longo dos anos. Para Francoeur et $a{ }^{3}$ os cirurgiões que começaram a videocirurgia tardiamente em sua carreira têm um índice maior de lesões. O treinamento adequado é capaz de melhorar o desempenho com ganho de destre$z^{22,23}$ e a experiência é fator fundamental para obtenção de bons resultados, mas não elimina completamente o risco de iatrogenias ${ }^{16,23,24}$.

Várias pesquisas foram iniciadas a partir dos relatos do aumento da incidência de lesões iatrogênicas da VBP e da dificuldade na transmissão do conhecimento e da técnica. Estes trabalhos tiveram como objetivo desenvolver métodos melhores de treinamento, estabelecer um 
número mínimo de procedimentos necessários para que um cirurgião pudesse ser considerado proficiente e, portanto, habilitado a realizar videocirurgia e avaliar a relação desta habilitação com o risco de iatrogênias ${ }^{25-27}$. Em que ponto da curva de aprendizado o cirurgião está apto a operar sem supervisão? Qual é o número de procedimentos necessários para que um cirurgião possa ser considerado habilitado a realizar videocirurgia? Qual a relação desta habilitação com o risco de iatrogênias?

Surgiram então cursos teórico-práticos em videocirurgia, com duração variável nos quais os cirurgiões recebem os rudimentos práticos da videocirurgia operando animais ou mesmo treinando em "caixa-preta". O objetivo destes cursos é encurtar a curva de aprendizado, mas as evidências da eficácia destes tipos de treinamento ainda são fracas. Os simuladores de realidade virtual têm conquistado espaço como método de treinamento do desenvolvimento psicomotor. Esta modalidade de treinamento também é alvo de críticas no sentido de que as tarefas realizadas são muito simples e em nada semelhantes à operação "in vivo" além de ser muito onerosa ${ }^{15,27-29}$. Datta et al ${ }^{22}$ afirmam que cirurgiões que tiveram em sua residência treinamento em videocirurgia tiveram um índice menor de lesões da VBP em sua prática afirmando que o treinamento regular durante a residência é mais eficaz do que cursos de curta duração ${ }^{30}$.

Nesta série em que foram avaliados 21 cirurgiões, $15(71,42 \%)$ participaram de algum tipo de treinamento em cursos ou simuladores. Destes, 20\% tiveram lesão da VBP. Entre os outros seis (28,58\%) cirurgiões que não fizeram cursos de treinamento, 33,33\% causaram lesões na VBP. Na análise simples desses dados pode-se dizer que embora a maior parte dos cirurgiões tenha feito algum tipo de curso de treinamento, este fato não os protegeu contra as lesões, porém a análise estatística mostrou que existe uma baixa associação entre cursos de treinamento e as lesões (coeficiente Phy=0,085415; coeficiente $P$ de Pearson=0,84215).

A forma mais fidedigna de se avaliar a aquisição e desenvolvimento das habilidades necessárias para que o cirurgião possa ser considerado proficiente em videocirurgia, ainda é controversa e, até o momento não há um consenso na literatura sobre os assunto ${ }^{31,32}$. Vários trabalhos foram publicados propondo várias formas de avaliação da proficiência do cirurgião, mas, muitos deles confundiam competência com proficiência. Competência é o que o indivíduo é capaz de fazer e proficiência é o que o indivíduo realmente faz. O grau de proficiência, isto é, o lugar que o cirurgião ocupa na curva de aprendizado, é avaliado de forma indireta, pela análise de parâmetros como a taxa de conversão, índice de complicações, permanência hospitalar e tempo operatório. Em muitas destas avaliações, apenas a velocidade com que o indivíduo realizava a tarefa era valorizada, e não a sua acurácia7, 16,17. Velocidade não é sinônimo de proficiência. Além disto, estes métodos de avaliação podem abrigar elementos subjetivos de análise, o que os torna passíveis de críticas e, de certa forma, inválidos $^{33}$.

Nesta série a média dos anos de experiência dos cirurgiões (tempo após a graduação) foi 11,61 anos com variação entre um e 29 anos. A média dos anos de prática dos cirurgiões do grupo A foi 21.6 variando entre três e 29 anos e a do grupo B foi 8,37 anos, variando entre um e 21 . Com exceção de um dos cirurgiões, todos os que tiveram lesão tinham mais de 20 anos de experiência na prática cirúrgica. A análise estatística mostrou que a média dos anos de experiência dos cirurgiões do grupo $\mathbf{A}$ (lesão) é significantemente maior que a do grupo B (sem lesão) $p=0,026983(p<0,05)$. As médias do número de CVL por ano e as médias do tempo de experiência em videocirurgia e suas associações com lesões da VBP não tiveram diferenças significativas; $p=0,31856$ e $p=0,30722$ respectivamente. Da mesma forma, a idade do cirurgião isoladamente não é um fator confiável para previsão de complicações ${ }^{33-}$ 35. A análise estatística mostrou que nesta série houve uma baixa associação entre a idade do cirurgião e as lesões da VBP (coeficiente $\mathrm{Phi}=0,027603$ e coeficiente $\mathrm{P}$ de Pearson=0,027592).

Em relação ao Certificado de Habilitação em Cirurgia Videocirurgia, dos 21 cirurgiões consultados, 11 $(52,38 \%)$ eram certificados e $10(47,62 \%)$ não. Dos cirurgiões certificados, três $(27,27 \%)$ tiveram lesões da VBP. A análise estatística revelou uma baixa associação entre o Certificado de Habilitação e as lesões (Phi=0,148003, Pearson=0,146408).

A ausência de lesões na fase inicial da curva de aprendizado dos cirurgiões desta série pode se dever à seleção, pela história clínica, de pacientes com doença biliar menos grave e, portanto, em teoria, mais fáceis de serem resolvidos, mesmo por cirurgiões iniciantes.

Segundo Connor ${ }^{11}$, 3/4 das lesões da VBP não são reconhecidas no momento em que são produzidas, mesmo quando o cirurgião já é experiente, e reafirma a hipótese de que o reconhecimento da anatomia é o principal fator de proteção quanto à lesão. Entre os fatores de risco para lesão, a identificação errada do ducto cístico foi assinalada em várias séries ${ }^{5,35,36}$. As variações da anatomia estão presentes em 6 a 25\% dos casos de lesão da VB de acordo com várias revisões bibliográficas ${ }^{21,37}$. Nesta série, as variações anatômicas estiveram associadas a 40\% das lesões.

Vários trabalhos demonstraram que a doença biliar no sexo masculino e no paciente idoso podem ser fatores de risco para lesões a VBP. Sabe-se que quanto mais longa é a história de doença biliar, maior é a possibilidade da presença de grande reação inflamatória local, com vesícula escleroatrófica, o que torna a operação mais difícil e com maiores riscos de lesão da VBP. A presença de vesícula escleroatrófica e colecistite crônica agudizada foi inesperada em dois casos. Nesta série a média de idade dos pacientes com lesão da VB foi 63,2 anos variando entre 34 e 80 anos. Os pacientes com lesões mais graves tinham 76 e 80 anos. 
O papel da colangiografia na prevenção das lesões ainda é discutível. Há os que afirmam que a colangiografia de rotina reduz a incidência de lesões ${ }^{16} \mathrm{e}$ os que acreditam que sirva mais para o diagnóstico precoce da lesão do que para preveni-la ${ }^{37}$. O exame rotineiro aumenta os custos e o tempo operatório e não é um procedimento completamente sem riscos ${ }^{38}$, mas tem a vantagem de prover ao cirurgião um estudo detalhado da VBP5. Várias séries, entretanto, mostraram que a lesão algumas vezes ocorre após a realização da colangiografia. Nesta série foi realizada em $66,66 \%$ dos casos em que houve lesão e mostrou-se muito útil no seu reconhecimento no mesmo ato operatório.

Considerando-se que os fatores de risco devam estar presentes no período de treinamento do que se convencionou chamar de primeira fase da curva de aprendizado, o que na prática nem sempre ocorre, o cirurgião proficiente deveria ser capaz de reconhecer a variação anatômica antes de lesar a VBP ou dissecá-la com segurança apesar do intenso processo infamatório. É questionável, assim, que se possa admitir uma curva de aprendizado padronizada baseada somente em número de procedimentos. Da mesma forma, o período de tempo que os cirurgiões levam para executar os primeiros casos de sua curva de aprendizado também parece ter importância na aquisição e sedimentação dessas novas habilida$\operatorname{des}^{37-39}$.

As lesões iatrogênicas da VBP que ocorreram nesta série, com cirurgiões já considerados experientes em $C V L$, poderiam sugerir o desenho de uma nova curva de aprendizado, para casos mais complexos.

Concluindo: o certificado de Habilitação, treinamento prévio em videocirurgia e a idade do cirurgião não se relacionaram com as taxas de lesão da VBP nesta série.

Houve significância estatística na diferença entre as médias dos tempos de experiência e sua relação com as lesões da VBP entre os cirurgiões que ocasionaram lesões da VBP e os cirurgiões que não tinham pacientes com este tipo de lesão.

\title{
A B S T R A C T
}

\begin{abstract}
Objective: the aim of this study is to analyze surgeon's proficiency, based on the different aspects of his learning curve as a risk factor for iatrogenic biliary tract injuries associated with Laparoscopic Cholecystectomy. Methods: a retrospective study was conducted using the report information from charts of patients January 1992 through December 2007; at Hospital Universitário Clementino Fraga Filho da Universidade Federal do Rio de Janeiro. Analysis from data collected from charts of 2285 patients who had undergone laparoscopic cholecystectomies. Data from surgeons involved with this type of surgery was also analyzed. Results: a total of six injuries $(0.26 \%)$ were found. This result is similar to that found in international publications. All injuries had occurred in the surgeon's second phase of the learning curve with more than 50 video surgeries. Conclusion: it was conclude that Video surgery Certificate and previous training are not related to biliary tract injuries in this series. Surgeon's age was not a risk factor in this study. There was statistical significance between surgeons' experience and injuries.
\end{abstract}

Key words: latrogenic disease. Laparoscopic cholecystectomy. Video-assisted surgery. Learning. Education.

\section{REFERÊNCIAS}

1. Csendes A, Navarrete C, Burdiles P, Yarmuch J. Treatment of common bile duct injuries during laparoscopic cholecystectomy: endoscopic and surgical management. World J Surg. 2001; 25(10):1346 -51.

2. McKenzie S, Schwartz R. The management of bile duct injuries occurring during laparoscopic cholecystectomy. Curr Surg. 2006; 63(1):20-3

3. Francoeur JR, Wiseman K, Buczkowski AK, Chung SW, Scudamore $\mathrm{CH}$. Surgeon's anonymous response after bile duct injury during cholecystectomy. Am J Surg. 2003; 185(5): 468-75.

4. Schmidt SC, Langrehr JM, Mintze RE, Neuhaus P. Long-term results and risk factors influencing outcome of major bile duct injury following cholecystectomy. Br J Surg. 2005; 92(1): 76-82.

5. Richardson MC, Bell G, Fullarton GM. Incidence and nature of bile duct injuries following laparoscopic cholecystectomy: an audit of 5913 cases Br J Surg. 1996; 83(10): 1356-60.

6. Morgenstern L, McGrath MF, Carroll BJ, Paz-Partlow M, Berci G. Continuing hazards of the learning curve in laparoscopic cholecystectomy. Am Surg. 1995; 61(10): 914-8.
7. Fletcher DR, Hobbs MS, Tan P, Valinsk LJ, Hockey RL, Pikora TJ et al. Complications of cholecystectomy: risks of the laparoscopic approach and protective effects of operative cholangiography: a population-based study. Ann Surg. 1999; 229(4): 449 -57.

8. Windsor JA, Pong J. Laparoscopic biliary injury: more than a learning curve problem. Aust N Z J Surg. 1998; 68(3): 186-9.

9. Lekawa M, Shapiro SJ, Gordon LA, Rothbart J, Hiatt JR. The laparoscopic learning curve. Surg Laparosc Endosc.1995; 5(6): 455-8.

10. Karvonen J, Gullichsen R, Laine S, Salminen P, Grönroos JM. Bile duct injuries during laparoscopic cholecystectomy: primary and long-term results from a single institution. Surg Endosc. 2007; 21(7):1069-73. Epub 2007 May 19.

11. Connor S, Garden OJ. Bile duct injury in the era of laparoscopic cholecystectomy. Br J Surg. 2006; 93(2):158-68.

12. SOBRACIL. Disponível em: secretaria@sobracil.org.br. Acesso em 23 de julho de 2008

13. Colégio Brasileiro de Cirurgiões. Disponível em: http:// www.cbc.org.br Acesso em 22 de junho de 2008.

14. Aguiar GB, Oliveira CIB, Silva Júnior JBS, Santos LS, Vieira SC. Lesão iatrogênica de vias biliares. Rev Col Bras Cir. 2005; 32(2): 6973. 
15. Hobbs MS, Mai Q, Knuiman MW, Fletcher DR, Ridout SC. Surgeon experience and trends in intraoperative complications in laparoscopic cholecystectomy. Br J Surg. 2006; 93(7): 844-53.

16. Misawa $T$, Saito $R$, Shiba $H$, Son $K$, Futagawa $Y$, Nojiri $T$ et al. Analysis of bile duct injuries (Stewart-Way classification) during laparoscopic cholecystectomy. J Hepatobiliary Pancreat Surg. 2006; 13(5): 427-34

17. Sequeira R, Weibaum F, Satterfield J, Chassin J, Mock L. Credentialing physicians for new technology: the physician's learning curve must not harm the patient. Am Surg. 1994; 60(11): 821-3.

18. Voitk AJ, Tsao SG, Ignatius S. The tail of learning curve for laparoscopic cholecystectomy. Am J Surg. 2001; 182(3): 250-3.

19. Cezário Melo MA. Questões relacionadas à aprendizagem motora na videocirurgia. Rev Bras Videocirurgia. 2007; 5(2): 72-89.

20. Cezário Melo MA. Curva de aprendizado na videocirurgia. Rev Bras Videocirurgia. 2004; 2(30):111-2.

21. Krähenbühl L, Sclabas G, Wente $M N$, Schäfer $M$, Schlumpf R, Büchler MW. Incidence, risck factors and prevention of biliary tract injuries during laparoscopic cholecystectomy in Switzerland. World J Surg. 2001; 25(10): 1325-30.

22. Datta V, Bann S, Aggarwal R, Mandalia M, Hance J, Darzi A. Technical skills examination for general surgical trainees. Br J Surg. 2006; 93(9): 1139-46.

23. Datta V, Chang A, Mackay S, Darzi A. The relationship between motion analysys and surgical technical assessments. Am J Surg. 2002; 184(1): 70-3.

24. Madureira Filho D. Técnicas avançadas de cirurgia laparoscópica. São Paulo: Atheneu: 2001

25. Anderson $\mathrm{Cl}$, Jentz $\mathrm{AB}$, Harkema JM, Kareti LR, Apelgren $\mathrm{KN}$, Slomski CA. Assessing the competencies in general surgery residency training. Am J Surg. 2005; 189(3): 288-92.

26. Sidhu RS, Grober ED, Musselman LJ, Reznick RK. Assessing competency in surgery: where to begin? Surgery. 2004; 135(1): 6-20.

27. Vassiliou MC, Feldman LS, Andrew CG, Bergman S, Leffondré K, Stanbridge D, Fried GM. A global assessment tool for evaluation of intraoperative laparoscopic skills. Am J Surg. 2005; 190(1):10713.

28. Risucci DA. Visual spatial perception and surgical competence. Am J Surg. 2002; 184(3): 291-5.

29. Hugh TB. New strategies to prevent laparoscopic bile duct injury surgeons can learn from pilots. Surgery. 2002; 132(5): 826-35.
30. Bann S, Khan M, Datta V, Darzi A. Surgical skill is predicted by the ability to detect errors. Am J Surg. 2005; 189(4): 412-5.

31. Risucci D, Geiss A, Gellman K, Pinard B, Rosser J. Surgeon-specific factors in the acquisition of laparoscopic surgical skills. Am J Surg. 2001; 181(4):289-93

32. Sawaki L, Yassen Z, Kopley L, Cohen LG. Age-dependent changes in the ability to encode a novel elementary motor memory. Ann Neurol. 2003; 53(4): 521-4.

33. Ribeiro M. Cirurgia minimamente invasiva. In: Neto GPB, Salles RARV, editores. PROACI - Programa de Atualização em Cirurgia. Ciclo2, Módulo 3. $1^{\circ}$ ed. Rio de Janeiro: Artmed; 2006 . p. 9-52.

34. Bingener J, Boyd T, Van Sickle K, Jung I, Saha A, Winston J et al. Randomized double-blinded trial investigation the impact of a curriculum focused on error recognition on laparoscopic suturing training. Am J Surg. 2008; 195(2):179-82.

35. Sawaki L, Yassen Z, Kopley L, Cohen LG. Age-dependent changes in the ability to encode a novel elementary motor memory. Ann Neurol. 2003; 53(4): 521-4.

36. Milcent M, Santos EG, Bravo Neto GP. Lesão iatrogênica da via biliar principal em colecistectomia videolaparoscópica. Rev Col Bras Cir. 2005; 32(6): 332-6.

37. Aggarwal R, Moorthy K, Darzi A. Laparoscopic skills training and assessment. Br J Surg. 2004; 91912):1549-58.

38. Waljee JF, Greenfeild LJ, Dimick JB, Birkmeyer JD. Surgeon age and operative mortality in the USA. Ann Surg.2006; 244(3): 35362.

39. Hamdorf JM, Hall JC. Acquiring surgical skills. Br J Surg. 2000; 87(1): 28-37.

Recebido em 26/02/2009

Aceito para publicação em 30/04/2009

Conflito de interesse: nenhum

Fonte de financiamento: nenhuma

\section{Como citar este artigo:}

Santos EG, Bravo Neto GP. Curva de aprendizado e lesões iatrogênicas em colecistectomias videolaparoscópicas. Rev Col Bras Cir. [periódico na Internet] 2010; 37(3). Disponível em URL: http://www.scielo.br/rcbc

\section{Endereço para correspondência:}

Elizabeth G. Santos.

Email: eligsant54@gmail.com 